Itinéraires Itinéraires

Littérature, textes, cultures

2013-2 | 2014

Sade et les femmes

\title{
Chantal Thomas lectrice de Sade
}

\section{Anne Coudreuse}

\section{(2) OpenEdition}

Journals

Édition électronique

URL : http://journals.openedition.org/itineraires/693

DOI : 10.4000/itineraires.693

ISSN : 2427-920X

Éditeur

Pléiade

Édition imprimée

Date de publication : 1 mars 2014

Pagination : 85-98

ISBN : 978-2-343-02712-8

ISSN : 2100-1340

Référence électronique

Anne Coudreuse, "Chantal Thomas lectrice de Sade », Itinéraires [En ligne], 2013-2 | 2014, mis en ligne le 01 novembre 2013, consulté le 10 décembre 2020. URL : http://journals.openedition.org/itineraires/ 693 ; DOI : https://doi.org/10.4000/itineraires.693

\section{$@ \oplus \Theta \Theta$}

Itinéraires est mis à disposition selon les termes de la licence Creative Commons Attribution - Pas d'Utilisation Commerciale - Pas de Modification 4.0 International. 


\section{Chantal Thomas lectrice de Sade}

\section{Résumé}

La lecture de Sade évolue-t-elle selon qu'on le lit d'une position universitaire ou du point de vue de la création romanesque? S'attachant à l'œuvre de Chantal Thomas, spécialiste reconnue de la littérature du XVIII ${ }^{e}$ siècle passée à l'écriture de romans historiques, la réflexion cherche à mettre en évidence une sorte de contestation possible d'une position par l'autre, comme si le roman révélait « l'impensé » d'une lecture plus universitaire de Sade.

Mots clés : réception, roman historique, libertinage, littérature du XVIII ${ }^{\mathrm{e}}$ siècle, création romanesque

\section{Abstract}

Does the way one reads Sade evolve according to whether one reads it from an academic standpoint or from the point of view of fictional creation? Focusing on the work of Chantal Thomas, a renowned specialist of the $18^{\text {th }}$ century who has turned to writing historical novels, this article attempts to show how each of these positions can, in a sense, challenge the other, as though the novel revealed the "unthought" side of a more academic reading of Sade's writings.

Keywords: reception, historical novel, libertinage, $18^{\text {th }}$ century literature, novel

Il est toujours inconfortable de proposer un commentaire sur l'œuvre d'un auteur vivant, surtout quand il s'agit d'une spécialiste reconnue de la littérature du XVIII ${ }^{\mathrm{e}}$ siècle, passée à l'écriture romanesque avec Les Adieux à la reine en 2002, puis Le Testament d'Olympe en $2010^{1}$. Il ne s'agit pas en effet de rendre compte de ce dernier roman dans un journal, mais de lire ensemble le travail de la romancière et celui de la critique qui fit paraître en 1978 Sade, l'œil de la lettre, repris en 2002, avec une préface inédite intitulée "Lire Sade », sous le titre Sade, la dissertation

1. Chantal Thomas, Le Testament d'Olympe, Paris, Seuil, 2010. Les renvois à ce roman sont indiqués entre parenthèses dans le texte. 
et l'orgie 2 , en référence à l'une des grandes scansions que Barthes voyait à l'œuvre dans l'écriture sadienne, telle qu'il l'analyse dans Sade, Fourier, Loyola ${ }^{3}$. Elle est aussi l'auteur du volume sur Sade de la collection « Écrivains de toujours » du Seuil paru en 1994. L'analyse que nous proposons prend le risque de l'erreur, du désaveu même, l'auteur contemporain ayant un droit de réponse, alors que le critique a toujours le dernier mot sur l'auteur mort... Mais ce risque confère à l'exercice toute sa saveur et toute sa délicatesse, une sorte de petite terreur qui fait que le jeu en vaut sans doute la chandelle. Il s'agira d'analyser les formes, les fonctions et les effets de l'intertextualité sadienne dans le roman de Chantal Thomas, pour mettre en évidence une forme de critique interne, qui est peut-être de l'ordre de l'impensé ou de l'inconscient: il nous semble en tout cas que Le Testament d'Olympe refuse l'amoralisme sadien et propose une lecture critique du libertinage, que les essais souvent enthousiastes de Chantal Thomas sur Sade étaient loin d'annoncer. Ce recul critique se fait au nom du refus d'une victimisation des femmes, à quoi semblent conduire l'univers romanesque et la pensée de Sade. Chantal Thomas présente une configuration singulière par rapport à d'autres femmes écrivains ayant écrit sur Sade ou à partir de lui. On peut rapprocher son roman de celui de Susan Sontag, L'Amant du volcan ${ }^{4}$, situé lui aussi au XVIII ${ }^{\mathrm{e}}$ siècle et faisant également la part belle à la très sadienne thématique volcanique, même si la romancière américaine n'a pas commenté l'œuvre de Sade d'un point de vue de spécialiste universitaire, comme l'a fait Chantal Thomas. Sa position diffère aussi de celle d'Annie Le Brun, auteur de Soudain un bloc d'abîme, Sade ${ }^{5}$, mais qui n'a jamais écrit de roman stricto sensu en rapport avec cet auteur, On n'enchaîne pas les volcans ${ }^{6}$ étant bien présenté comme un recueil d'essais en couverture. On verra en conclusion comment la démarche de Chantal Thomas diffère de celle d'Angela Carter qui se situe comme elle dans la double position de critique de Sade et de romancière, mais trente ans avant, et dans un contexte marqué par un féminisme beaucoup plus militant. Même si Chantal Thomas s'inscrit dans une constellation de femmes écrivains inspirées par Sade et son univers, il semble que le rapport entre la lecture critique qu'elle a faite de ses livres et sa création romanesque et ce qui s'y rejoue ou s'y déplace de cette lecture, se donne à penser comme un ensemble à la fois original, singulier et déstabilisant, à l'image même de l'œuvre sadienne peut-être.

2. Chantal Thomas, Sade, la dissertation et l'orgie, Paris, Payot et Rivages, 2002.

3. Roland Barthes, Sade, Fourier, Loyola [1971], « La dissertation, la scène », dans CEuvres complètes, Paris, Seuil, 1994, p. 1144.

4. Susan Sontag, L'Amant du volcan [The Volcano lover, 1992], trad. Sophie Bastide-Foltz, Paris, Christian Bourgois, 1995, rééd., coll. « Titres », 2011.

5. Annie Le Brun, Soudain un bloc d'abîme, Sade : introduction aux æeuvres complètes, Paris, Pauvert, 1986.

6. Annie Le Brun, On n'enchaîne pas les volcans, Paris, Gallimard, 2006. 
L'emprunt le plus évident à Sade dans Le Testament d'Olympe est d'ordre structurel : l'opposition entre deux sœurs, Apolline ${ }^{7}$ qui choisira le chemin de la vertu, et Ursule, qui sous le nom d'Olympe, prendra celui du vice. On reconnaît là sans peine le couple formé par Justine et Juliette chez Sade. Le roman de Chantal Thomas joue malicieusement sur le contraste entre les deux sœurs, dans des portraits qui sont autant de références à ceux de l'écriture sadienne :

Ursule avait les yeux noirs et les cheveux blond-roux; moi, les yeux clairs et les cheveux châtain foncé presque bruns, comme s'il s'était produit entre nous une inversion ou une confusion dans la répartition de nos attributs physiques et que ces yeux bleus qu'elle me disait d'admirer auraient dû être les siens. (p. 25)

Les choix narratifs permettent de faire entendre deux récits à la première personne du singulier, puisque, mariée et sauvée de la misère par ce mariage, Apolline se voit confier « plusieurs cahiers cousus à la main et aux feuilles noircies d'une écriture serrée » (p. 122), dont le contenu est révélé dès le chapitre suivant, "J'ai connu des prisons dorés ", où Olympe fait le récit de son ascension sociale qu'elle doit, comme Juliette chez Sade, à l'usage de ses charmes féminins. Dès le début du roman, pendant son enfance et ses années d'apprentissage, elle montre un savoir bien plus grand sur la question que celui de sa sœur qui n'y comprend rien, même quand Ursule cherche à lui expliquer le sens d'un bon mot scandaleux du maréchal de Richelieu :

Lors d'une audience, des bourgeois avaient réclamé auprès de Richelieu qu'il fît chasser de la ville les catins qui l'infestaient. Il faudrait donc, avait répondu Richelieu, que j'en chasse toutes les femmes.

«"Catin", lui ai-je demandé, qu'est-ce que ça veut dire? C'est lié à "câlin"?

- Exactement. La catin est une femme qui prodigue des câlins dont aucun n'est gratuit. »

Ça ne m'éclairait pas. (p. 24)

Plus loin dans le roman, Olympe se dit « déterminée à ne perdre [s]on pucelage qu'au prix fort » (p. 146). Le choix de ce prénom qui donne son titre au roman renvoie bien évidemment au personnage d'Olympe Borghèse, dans l'Histoire de Juliette, précipitée dans le Vésuve par son ancienne maîtresse et complice ${ }^{8}$. L'imaginaire du volcan, si prégnant dans

7. Le prénom Apolline est peut-être aussi une référence à Apollinaire, auteur des Onze mille verges (1907) et de L'CEuvre du marquis de Sade (1909).

8. Voir Sade, Histoire de Juilette, dans Euvres, éd. Michel Delon, Paris, Gallimard, coll. « Bibliothèque de la Pléiade », t. III, 1998, p. 1102. La spécialiste du XVIII e siècle qu'est Chantal Thomas fait peut-être aussi référence, par le choix de ce prénom, à Olympe de Gouges, considérée comme une des premières « féministes ", même si le mot n'existe pas encore au moment où elle défend la cause des femmes. 
l'univers de Sade 9 , se retrouve dans le roman de Chantal Thomas. Ursule qui deviendra Olympe a en effet " l'impression d'avoir un lampion à la place du sexe » (p. 130). Elle se moque du commentaire d' " un homme de sciences qui avait étudié avec M. de Buffon » à qui elle en a parlé :

Je ferais mieux d'aller consulter un vulcanologue, un spécialiste des volcans, a-t-il traduit pour me mettre à l'aise. L'idiot ! J'avais l'âme rebelle, la voix chaude. Le feu qui m'habitait allait et venait, il n'avait pas de lieu précis, j'avais dit le sexe comme ça, j'aurais pu dire aussi bien la nuque ou les oreilles. (p. 130)

Il n'est guère étonnant de voir revenir sous la plume de la romancière des images et un thème auxquels elle avait déjà été sensible dans sa lecture critique de Sade :

Le feu du ciel ne fait qu'un avec celui de la terre, l'orage perpétue l'irruption volcanique. Olympe jetée au fond d'un volcan, Justine foudroyée reçoivent des éléments de l'univers libertin le signe de leur exclusion, le geste de leur mort; alors que le libertin en reçoit l'approbation et la confirmation de ses plus terribles excès.

La foudre qui tue le corps vertueux se répand, sur celui qui s'ouvre au gouffre de la jouissance, en pluie de foutre ${ }^{10}$.

Une des étapes pour Apolline sera le couvent de Notre-Dame-de-la-Miséricorde, « à Libarre, au bord de la Garonne » (p. 45), ce qui rappelle bien sûr, pour Justine, le couvent de Sainte-Marie-des-Bois - jusque dans le mimétisme ironique des traits d'union entre les différents mots de ce nom religieux - et celui de Panthemont pour Juliette. Même si les pensionnaires du couvent d'Apolline ne se livrent pas aux mêmes débauches que celles qui sont décrites dans les couvents sadiens, on y trouve pourtant un éloge du parricide dans la bouche d'une de ses coreligionnaires :

«- Je devrais confesser les rêves où j'assassine M. de Terville! » [...]

«Que faites-vous à votre père? dis-je d'une voix presque inaudible.

9. «L'embouchure du volcan devient un sexe monstrueux qui engloutit ses victimes, elle proclame la vérité de la nature et de l'homme. Dans les romans contemporains, elle est métaphore des secrets de l'individu qui monte au sommet de l'Etna ou du Vésuve, comme on descend dans les profondeurs de sa mémoire ou de son inconscient. Dans les traités de poétique et d'esthétique, elle est l'image du sublime qui perturbe la distinction du beau et du laid, du locus ameoenus et du locus horribilis. Tous ces thèmes se réunissent pour faire du volcan l'image même de la nature aussi bien que de la fiction sadienne. Olympe Borghèse, qui ne peut prévoir sa fin, et Juliette se plaisent à imaginer l'univers comme "un volcan, dont d'exécrables forfaits jailliraient à chaque minute" (p. 838)» (Michel Delon, notice d'Histoire de Juliette, dans Sade, Euvres, op. cit., t. III, p. 1370). Cet imaginaire du volcan est également présent dans le roman de Susan Sontag, L'Amant du volcan. Il s'agit là aussi de l'œuvre d'une universitaire et essayiste adoptant la forme romanesque, dans un roman d'amour historique situé à Naples au XVIII ${ }^{\mathrm{e}}$ siècle. Or Susan Sontag était aussi connue pour son engagement en faveur du féminisme.

10. Chantal Thomas, Sade, la dissertation et l'orgie, op. cit., p. 53. 
- C'est selon l'arme que j'ai sous la main. Un couteau, une épée, un pistolet, du poison... Parfois il a le temps de se sentir mourir. Ses yeux durs deviennent suppliants, s'emplissent de larmes immondes. Il appelle au secours. Personne ne vient, j'ai enfermé tous les domestiques ensemble dans la cave... Il y a aussi la hache. Mon père est au lit avec Catherine, la servante. Je me penche sur lui, sur eux, et je lui tranche la tête. À lui seul. À chaque fois j'ai peur de décapiter aussi Catherine. Je fais bien attention.

- Taisez-vous! Comment osez-vous juger votre père?

- Je ne le juge pas. Je le tue.

— Mais, Mathilde, c'est un péché mortel de tuer. En plus, tuer son père...

- Tout est péché, Apolline. Ignorez-vous que nous n'avons pas le droit de nous raconter nos rêves...

- [...] ni nos confessions, ni nos pénitences, ni les actions des sœurs, ni ce que nous entendons au parloir, ni ce que nous observons au réfectoire. Je sais. » (p. 54-55)

Ce récit de rêve est évidemment une référence à la dissertation sur le parricide que demande Olympe à Juliette avant d'assassiner son père, pour lui en démontrer vertueusement toute l'horreur ${ }^{11}$. Chantal Thomas commente cette dissertation de Juliette pour Olympe Borghèse en ces termes dans son essai : «Ce discours, qui ne représente nullement l'expression (ni vraie ni fausse) de sa pensée, correspond plutôt au mouvement léger, immédiat par lequel on vient se prêter à une posture ${ }^{12} »$. Qui sait alors si cet éloge du parricide dans la bouche de Mathilde de Terville n'est pas une autre «posture », une sorte de pendant ironique à la critique qu'en a donnée Juliette?

En schématisant, on peut remarquer que Chantal Thomas développe, dans les portraits des libertins de son roman, deux caractéristiques que l'on trouve dans les romans sadiens : 1'apathie et le rire ${ }^{13}$. " Le rire dans Sade, note-t-elle dans son analyse, appartient sans partage à la caste des libertins. De la pointe légère d'un éclat de terreur, il déchire sans effort le règne du sérieux et de la vraisemblance ${ }^{14} »$. Il est interdit aux victimes qui «l'utiliseraient en dénégation de l'ordre du libertinage, comme moyen de s'en protéger ou de s'accommoder de sa rigueur ${ }^{15} \gg$. Elle illustre dans son roman un trait de l'univers sadien qu'elle a très bien analysé dans son essai :

Mais aux larmoiements, jérémiades et supplications des victimes, le sacrificateur répond par un éclat de rire $[\ldots]$ : irréversible est la dynamique de jouissance dont elles vont faire les frais, et les raisons qu'elles pourraient tenter de lui opposer sont pures risées ${ }^{16}$.

11. Sade, Histoire de Juliette, dans Euvres, op. cit., t. III, p. 811-812.

12. Chantal Thomas, Sade, la dissertation et l'orgie, op. cit., p. 72.

13. Sur la question de l'apathie, voir Anne Coudreuse, Le Refus du pathos au XVIII siècle, Paris, Champion, 2001, p. 227-236.

14. Chantal Thomas, Sade, la dissertation et l'orgie, op. cit., p. 68.

15. Ibid., p. 69.

16. Ibid. 
Toutes ces idées se trouvent développées de façon romanesque lors du premier entretien d'Olympe et de Richelieu, à propos de « ce pauvre Rulhière », qui l'a recueillie, et qui est « consumé par [s]a passion » pour la comtesse d'Egmont :

Je m'esclaffais.

«Mourir d'amour, quelle bêtise!

- Cela te fait rire! Tu ne rêves pas amour, billets doux, serments au clair de lune?

— Nullement! Aimer rend faible et ridicule. Une fille amoureuse n'est qu'une loque.

— Quelle âme sympathique! Olympe, j'ai du plaisir à t'écouter. Tes déclarations me changent des habituelles fadaises de ton sexe.» (p. 166)

On retrouve ce sens de la dérision chez Louis $\mathrm{XV}$, grande figure de libertin dans le roman, dont se plaint Olympe à l'abbé Delbès, nom qui évoque sans doute ironiquement celui de Delbène, la supérieure de l'abbaye de Panthemont, qui dans l'itinéraire initiatique de Juliette, la fait bénéficier d'un savoir philosophique, tandis que Clairwil lui livre un savoir social et Durand, la sorcière, un savoir technique et magique. Le roi Louis XV a fait un enfant à Olympe et l'a laissé mourir sans soins, en exilant sa mère, pour ne pas déplaire à la Pompadour. Cette accusation scandalise l'abbé :

Je vous en prie, madame, vous blasphémez. Songez qui vous osez accuser. Sa Majesté est roi de droit divin. Il a été désigné par le Très Haut, dit-il en se signant. (Je me souvins du rire de Louis XV : « Je suis l'oint du Seigneur, Richelieu, ne l'oubliez pas », de la lumière sur ses bagues, de sa main entre mes seins.) (p. 287)

L'apathie pour sa part se manifeste par un refus de s'attacher à qui que ce soit, ce qui est une «calamité intérieure 》 (p. 237) comme en témoigne Ursule, dès la première partie du roman, quand sa sœur lui demande si elle est « amoureuse ». Elle la toise alors «d'un air glacial», et lui répond : «Je n'aime personne, Apolline. Tu n'as pas compris?» (p. 42). Alors qu'une voyante lui a annoncé : « Vous approcherez la grandeur et serez capable de la perpétuer » (p. 194), Olympe préfère mettre de côté cette prédiction : «Elle me faisait tourner la tête. Et je tenais à la garder froide » (p. 195). Il s'agit là d'une expression et d'une valeur typiques des libertins sadiens. Le bréviaire de Richelieu tient en ces mots : « Ne pas souffrir, dormir beaucoup, vivre toujours » (p. 193), ce qu'Olympe commente ainsi : "L'amour n'occupait aucune place dans le cœur de Richelieu. Dans son emploi du temps beaucoup, dans ses émotions rien » (Ibid.). Il ne se reconnaît « aucune dette, ni morale, ni d'argent» (p. 154), ce qui le rapproche également de Don Juan, libertin exemplaire. L'apathie de Richelieu semble même être le secret paradoxal de sa séduction : «Il avait ce charme : alors qu'il n'éprouvait rien, il donnait follement envie de l'aimer. Ou bien était-ce parce qu'il n'éprouvait rien qu'il donnait envie de l'aimer » (p. 184). Chantal Thomas opère une réécriture en réduction 
de la perte de la présidente de Tourvel par le vicomte de Valmont dans Les Liaisons dangereuses. La victime de Richelieu s'appelle Mme Michelin, et il manifeste envers elle un cynisme sans faille (p. 168-169). Poursuivant la piste de Baudelaire qui voyait en Mme de Tourvel une bourgeoise, ce qui permettrait de lire le roman de Laclos dans sa dimension de guerre sociale, Chantal Thomas fait de Mme Michelin «l'épouse d'un miroitier » (p. 167). Cherchant à séduire cette " céleste dévote », le duc de Richelieu se justifie ainsi : «il faut tâter aussi de la bourgeoise » (p. 168). La conquête dure plusieurs mois, comme dans le roman de Laclos, mais tient en quelques lignes dans le sommaire de ce récit inséré, ce qui augmente peut-être le cynisme du libertin Richelieu :

Plus d'une semaine pour se rapprocher d'elle à la messe, encore une dizaine de jours pour qu'elle prête l'oreille à ses déclarations, un long mois pour qu'elle capitule intérieurement, un mois encore plus long pour qu'elle accepte de le reconnaître. Alors, fou d'excitation, il l'avait emmenée dans un appartement loué exprès pour elle, près de l'église Saint-Paul, et avait tout obtenu. (p. 168)

Il raconte qu' « elle est morte » après qu'il l'a abandonnée et à ce récit « ses yeux étaient plissés d'une hilarité contenue» (p. 169), car c'est le veuf lui-même qui lui a appris cette funeste nouvelle, en ajoutant, avec une ironie tragique dans laquelle Richelieu voit « du Molière » : "Vous seul auriez pu la sauver. [...] Même au plus profond de sa mélancolie elle allait toujours mieux lorsqu'elle vous avait vu » (p. 169). Le modèle secret qui permet de composer cette figure de libertin dans les différents épisodes de sa carrière, c'est bien le vicomte de Valmont des Liaisons dangereuses.

Le roi lui-même se caractérise avec les mêmes mots que ceux de l'ogre Minski, grande figure de libertin à qui Sade fait dire dans l'Histoire de Juliette : "Mon cœur se blase, je ne jouis plus ${ }^{17}$ ». Alors qu'Olympe réclame «le cordon bleu du Saint-Esprit» pour son fils Louis Aimé, Louis XV son père se fâche :

- Craignez, Olympe, qu'à cause de vos importunités ce nourrisson ne soit par mon ordre décoré de l'ordre du Calvaire. À vingt-sept ans j'étais père d'une famille de dix enfants. Vous trouverez bon qu'à l'âge de cinquante et un ans, pour un énième rejeton, né de surcroît hors les liens sacrés du mariage, je n'entre pas en délire. J'ajoute qu'à l'âge de cinq ans j'étais déjà le père des vingt-deux ou vingt-trois millions d'âmes que compte mon royaume. Cela vous blase ${ }^{18}$. (p. 246)

17. Cité par Chantal Thomas, dans Sade, Paris, Seuil, coll. «Écrivains de toujours », 1994, p. 153. Sade, Histoire de Juliette, op. cit., p. 718.

18. Voir Chantal Thomas, Sade, la dissertation et l'orgie, op. cit., p. 35 : « Numériquement parlant les personnages sadiens se définissent toujours par un chiffre, celui de leur âge. Il indique la valeur essentielle en matière de libertinage : la dépravation de l'esprit. Et celleci, nullement cornélienne en cela, ne peut ignorer le nombre des années. Car elle est faite d'une suite de répugnances vaincues et maintenues à la fois, la jouissance dernière étant 
Les libertins sadiens se définissent aussi par une cruauté bien caractéristique dont on retrouve de nombreuses traces dans Le Testament d'Olympe. C'est ainsi que Zéphyrine, compagne d'Olympe qui veut faire une carrière au théâtre, se plaint de Richelieu : «Et il a cette manie de me mordre la langue jusqu'au sang! » (p. 176). Cette cruauté est également sociale, et on trouve de nombreux exemples de la morgue nobiliaire des libertins, comme la façon dont Richelieu parle des paysans : «Ah, les fripons! Je foutrai tout ça dans un cul-de-basse-fosse!» (p. 158), ou tout son mépris pour la bourgeoise Mme Michelin :

Les bourgeois ont ce travers, Olympe, ils se prennent pour quelque chose. Ils croient qu'il s'agit de vouloir, de faire des efforts, de fournir des preuves. Pas du tout. La vérité est simple. La langue parle d'elle-même. Vous êtes né ou vous ne l'êtes pas. (p. 171)

Richelieu forme Olympe à l' «école du mépris » (p. 172), non sans violence quand elle se montre rétive devant tant de cynisme : " Il me devina, posa sa main sur ma nuque et la serra avec la dureté d'un étau. Il pouvait me briser » (ibid.). C'est pourtant bien un principe de dégénérescence qui semble caractériser les libertins dans le roman de Chantal Thomas. Ils apparaissent à Olympe dans leur « ignoble vérité » (p. 251). Le duc de Richelieu est un " bouffon comparé à son ancêtre le cardinal », et Louis XV n'est qu' " un déchet de royauté », " un néant comparé à son bisaïeul Louis XIV » (ibid.). Olympe, formée à l'école d'un libertinage sadien, proclame son athéisme et son refus du providentialisme et d'une nature bienfaisante (p. 286). Les scènes sadiennes proprement dites sont rares ou inexistantes. En effet Sade procède par accumulation, excès, détails ${ }^{19}$, quand Chantal Thomas s'en tient le plus souvent dans son roman à l'allusion, ou du moins ne s'étend jamais bien longtemps dans l'écriture pornographique. Les jeux sadiens des valets de Mme de Vintimille avec son cadavre n'occupent qu'un paragraphe (p. 230). Olympe n'est fouettée qu'une fois (p. 205-206), alors que la fustigation est une pratique récurrente dans les romans sadiens. Le lecteur trouvera certes une scène libertine (p. 151-152), mais l'écriture en est plus proche de celle, «gazée », des Infortunes de la vertu, que de la pornographie de La Nouvelle Justine ou de l'Histoire de Juliette. Bien souvent, la dimension sadique ou sadienne du roman de Chantal Thomas et de ses personnages s'exprime par le biais de fantasmes, alors que la caractéristique essentielle des libertins de Sade est bien le passage à l'acte. Olympe exprime ainsi son désir cruel de déshabiller la Pompadour, puis, après l'avoir ligotée à une colonne, de lui ôter

d'être soi-même objet de répulsion pour les autres. Il n'est pas de jeune libertin; c'est une contradiction dans les termes $»$.

19. Ibid., p. 84 : « Les Prospérités du vice se jouent sur la scène essentiellement baroque du langage. Elles manifestent, au-delà des besoins de la communication, les richesses illimitées d'un style». 
avec une pince « un à un les poils du pubis » (p. 229). Juliette et Clairwil sont plus expéditives avant de jeter Olympe Borghèse dans le Vésuve : « Nous épilâmes sa motte ${ }^{20}$ ». Dans l'ennui du couvent, Apolline invente le personnage de Café « devenue chef d'une bande de faux-monnayeurs » (p. 60), ce qui renvoie évidemment au personnage de Roland dans les aventures de Justine. La féminisation du personnage montre qu'il peut fonctionner comme une sorte de double de Juliette. Café a en effet à sa disposition « un équipage uniquement constitué de femmes [...] prêtes à mourir pour elle ou à tuer avec férocité selon ses ordres » (p. 61), ce qui n'étonne guère quand on sait que Chantal Thomas intitule "Les tribades » le chapitre qu'elle consacre à l'Histoire de Juliette dans sa présentation générale de l'œuvre de Sade ${ }^{21}$. C'est à la faveur d'un incendie détruisant le couvent qu'Apolline peut le quitter (p. 81-82). Là encore, la référence aux aventures de Justine est évidente, mais sur le mode allusif, humoristique pour tout dire. « Mère Lamproie [...] vociférait sa haine du pompier $[\ldots]$ : arrachez vos membres poilus de nos corps de viergezimmmaculées, mâlezimmmondes! » (p. 82). La dimension tragique de l'incendie dont est accusée Justine chez Sade est totalement gommée ici, et n'existe plus qu'à l'état de souvenir intertextuel.

Les Cent Vingt Journées de Sodome constituent un autre intertexte discret, dans la description du paysage de l'exil d'Olympe, qui évoque les abords du château de Silling (p. 268), y compris avec la neige qui tombe (p. 271) à laquelle Chantal Thomas se montrait déjà attentive dans son premier essai :

Les bruits du monde sont effacés, les cris des victimes étouffés : de la neige à l'entour du château de Silling, de l'ouate dans la gorge des suppliciés. Le silence est total qui enveloppe des «crimes sourds». Le crime sadien est également sourd aux clameurs extérieures (cris des victimes, répression de la société) et à sa propre voix. Cette complète surdité s'oppose à l'écoute constante et attentive qui est exigée des victimes ${ }^{22}$.

Ceci étant posé, on peut se demander si certains mariages ne sont pas des reproductions miniatures du château de Silling, en particulier celui de Mme de V., à Semoule :

M. de V. était un homme cruel. Le principal usage qu'il avait de son épouse - comme me l'apprirent rapidement les domestiques - était de la battre. [...] Au premier regard entre eux, il ne l'avait jugée bonne qu'à souffrir. Mme de V. s'était soumise sans restriction. Elle s'était vouée à endurer tout ce qui lui viendrait de cet homme. (p. 87)

20. Sade, Histoire de Juliette, op. cit., p. 1102.

21. Chantal Thomas, Sade, op. cit., p. 147-159.

22. Chantal Thomas, Sade, la dissertation et l'orgie, op. cit., p. 45. 
Pour Chantal Thomas, «la femme-épouse [...] illustre chez Sade le dernier degré victimal auquel un être puisse atteindre ${ }^{23} »$. Selon ce principe, c'est Apolline qui serait la plus à plaindre, puisqu'elle se marie à Arnaud de Terville et le suit en Angleterre : « Sûre de moi comme de lui. [...] Ses lèvres avaient un goût de fruit » (p. 122). La construction narrative du roman en diptyque, introduit par une note de juillet 1771 rédigée par Apolline à Londres, produit un effet de sens évident : les derniers mots du roman sont un réquisitoire contre le roi, figure de libertin et objet de blâme. Le roman culmine donc dans ce refus de l'amoralisme et de l'inconséquence propres aux personnages emblématiques du libertinage chez Sade :

Et vous, assis au bout du lit, sur votre trône magnifique, les bras posés sur les accoudoirs, le buste penché pour mieux nous observer et comparer les avantages, les types de beauté, l'embonpoint de la gorge, la rondeur de la cuisse, la blancheur de la peau, l'incarnat de nos joues, les perles de nos dents, la fleur de notre virginité, vous possédez deux couronnes [...]. Je profiterai de la situation jusqu'au bout, je ne compte pas les folles qui périront à ma place, et je le proclamerai à la face du monde, dans tous les châteaux et les hôpitaux, j'assourdirai le ciel et la terre, je l'écrirai partout et pour toujours, Moi, Olympe Ursule Aubain Bourbon de Quizière, née de Pauvrelieu, ai été assassinée sur ordre du roi, de lâche et épouvantable façon. (p. 302-303)

Le mot de la fin vient donc comme une condamnation, annoncée déjà dans le roman par les descriptions de la dégradation physique de Zéphyrine (p. 296) et d'Olympe, notamment lors du séjour de cette dernière à la Salpêtrière et à Bicêtre. C'est à ce prix que les libertins se divertissent. Leur libertinage a des conséquences sociales et physiques très dégradantes pour les femmes qui sont leurs victimes. Même si pour Chantal Thomas, il s'agit d'un critère réversible, qui ne fait pas vraiment une différence entre le libertin et la femme qu'il asservit ${ }^{24}$, tout porte à penser, dans l'agencement du roman, qu'elle condamne le sort fait aux femmes dans l'univers sans morale du libertinage qui les nie. C'est dans « un immeuble lépreux » qu'Apolline retrouve Olympe : «Une femme squelettique [...] à peu près nue sous un haillon » (p. 102). Dans ce passage au registre pathétique assumé, se lit en filigrane une critique des conséquences du libertinage qui est précisément un refus des conséquences :

Je me jetai sur son corps dévasté, sanglotai contre sa poitrine, l'embrassai, je voulais la réchauffer de mon amour, la ranimer, la sauver. Quand je me

23. Ibid., p. 90.

24. Ibid., p. 74-75 : «Sade n'a rien à voir avec une problématique du maître et de l'esclave. Le désir d'être victime, tout autant que celui de "victimer", désigne le libertin. Et la demande: "Jure-moi qu'un jour aussi je serai ta victime" est immédiatement reconnue comme une parole de maître, comme l'indice admirable d'une très profonde et infléchissable souveraineté ». 
suis relevée, Ursule avait cessé de vivre. Elle avait perdu sa guerre. J'étais comme happée par son profil maigre, à jamais figée par la mort. (p. 103)

On peut risquer cette hypothèse d'interprétation: en mode fantasmatique, le libertinage sadien est un réservoir de scènes, de situations, de dialogues qui sert une écriture enlevée, presque toujours au second degré et illustre « la souveraine liberté de l'imaginaire », pour reprendre une expression de Michel Delon ${ }^{25}$. Est ainsi décrit, par exemple, le régime du libertin Richelieu pour être «le plus vert», et qui est, "à plus de soixante ans [...] un maître gamahucheur » (p. 248). En mode réaliste au contraire, quand le lecteur est confronté à la misère sociale et physique de ses victimes, ce libertinage fait l'objet d'une condamnation qui semble difficilement contestable, dans des descriptions saisissantes, à lire cette fois au premier degré, non sans un frisson de dégoût ou d'horreur. La confession d'Olympe commence par la fin :

Je suis passée au grand remède, section de la Force, salle de la Miséricorde. Le grand remède commence par le fouet. Il se termine par la mort. J'allais être transportée à Bicêtre, un homme m'a prise chez lui. Il a eu envie de ce squelette seulement pour lui.

À la Salpêtrière, la « grande maison » comme on dit, les officières nous frappent, nous volent la nourriture. (p. 123-124)

Le ton est proche ici de l'empathie qui caractérise le récit de Marie Didier, Dans la nuit de Bicêtre ${ }^{26}$. Pour corroborer cette hypothèse d'un double mode de l'intertextualité sadienne dans le roman, l'un fantasmatique, l'autre réaliste, un détour par l'écriture autobiographique est peut-être nécessaire, auquel Chantal Thomas semble elle-même nous autoriser, puisqu'elle propose quelques aperçus sur sa jeunesse dans la dernière partie de son essai intitulée «Juliette, ô Juliette». La lecture de Sade semble être le seul espace de liberté dans l'univers carcéral du lycée :
Lire d'enfermement
Un dortoir n'est pas un foutoir. Il faut savoir s'endormir à heures fixes. [...] Bordeaux. Lettres supérieures. Une année de prison dans l'enveloppement gris, lourd et doux d'une catastrophe indéfiniment retenue, déjà survenue. Port mort. Se débattre, c'est s'enfoncer. [...] Les auteurs au programme ça sonne à ses oreilles comme le régime des colonels. Sur un même fond de pas cadencés, d'exécutions secrètes ${ }^{27}$.

Cet appendice se termine par deux pages splendides d'autobiographie cryptée qui ne livrent rien de leurs secrets mais en préservent intacte l'incandescence exacte. Faute de pouvoir les citer intégralement, nous en donnons un extrait qui témoigne de décrochements énonciatifs

25. Michel Delon, "Histoire de Juliette », dans Jean-Pierre de Beaumarchais et Daniel Couty (dir.), Dictionnaire des æuvres littéraires de langue française, Paris, Bordas, 1994.

26. Marie Didier, Dans la nuit de Bicêtre, Paris, Gallimard, 2006.

27. Chantal Thomas, Sade, la dissertation et l'orgie, op. cit., p. 161-163. 
bouleversants, comme une manière de danser autour de l'autobiographie dans la peur de s'y brûler :
J'adore le rouge, tu me le tends, œuf de porphyre, volcan ovale et lisse, au creux de ma paume, quelle fraicheur, tu perds pied, et c'est pourpre.
La lettre découpée
Un jour, elle reçoit dans une enveloppe trop grande une carte rectangulaire.
[...] C'est une carte de faire-part dont on a découpé la bande noire, le cerne de deuil ${ }^{28}$.

Il n'est sans doute pas anodin que les deux sœurs du roman soient originaires de Bordeaux, comme une manière de boucler la boucle en l'ouvrant infiniment...

Notre lecture de Chantal Thomas, cette lectrice de Sade passée à une écriture romanesque où l'intertextualité sadienne joue un rôle primordial, met donc en évidence une forme de critique interne, dans la création romanesque, des analyses proposées dans le discours théorique. La romancière puise dans un imaginaire sadien qu'elle connaît parfaitement du fait de tout le travail analytique qu'elle a mené sur ses éléments, sans approuver pour autant les effets qu'il entraîne dans le réel. Tout se passe comme s'il $\mathrm{y}$ avait une pensée du roman, se présentant comme une manière de penser contre soi, ou à côté, de laisser advenir un insu de son savoir théorique et critique d'une œuvre unique en son genre, dont Chantal Thomas écrit : « Ni le savoir que j'ai acquis depuis [cette découverte], ni une sorte d'officialisation de Sade n'ont banalisé sa lecture. Ce bouleversement, premier, cette brûlure, restent intacts ${ }^{29}$ ». C'est peut-être de cette brûlure que Chantal Thomas a essayé d'explorer la nature, en changeant d'angle et en passant de la position critique à l'écriture créatrice, non sans susciter un certain nombre de brouillages entre les deux. Ce qui semble persister d'une position à l'autre, c'est l'intérêt pour le thème de la liberté, défendue aussi bien du point de vue de l'analyse que dans la création romanesque, dans une écriture caractérisée dans son versant critique comme dans son versant créatif par «le principe de délicatesse », expression sadienne reprise par Michel Delon dans sa plus récente étude sur le libertinage au XVIII ${ }^{\mathrm{e}}$ siècle $^{30}$.

28. Ibid., p. 183.

29. Ibid., Quatrième de couverture.

30. Michel Delon, Le Principe de délicatesse. Libertinage et mélancolie au XVIII ${ }^{e}$ siècle, Paris, Albin Michel, 2011. Il a trouvé cette formule dans une lettre de Sade à sa femme, quand il est enfermé à la Bastille, durant l'hiver 1783 : «Voulez-vous mon linge sale, mon vieux linge? Vous voyez que je sens le prix des choses. Écoutez, mon Ange, j'ai toute l'envie du monde de vous satisfaire sur cela, car vous savez que je respecte les goûts, les fantaisies, quelque baroques qu'elles soient, je les trouve toutes respectables, et parce que l'on n'en est pas le maître, et parce que la plus singulière et la plus bizarre de toutes, bien analysée remonte toujours à un principe de délicatesse » (cité p. 15). C'est aussi à une lettre de Sade à sa femme que Marianne Alphant emprunte le titre qu'elle donne à son évocation 
Le rapport entre la position critique sur l'œuvre de Sade et la démarche créatrice semble plus complexe et moins illustrative dans le parcours de Chantal Thomas que dans celui d'Angela Carter, qui a publié la même année, 1979, The Sadeian Woman, une lecture féministe de la pornographie sadienne, et The Bloody Chamber, une réécriture des Contes de Perrault selon une perspective également féministe et dans le sens d'une libération de la femme, au centre des débats et des combats dans les années 1970. Publié à Londres par Virago, une maison d'édition féministe, The Sadeian Woman, présenté comme " an exercise in cultural history », a été traduit en français la même année ${ }^{31}$. Selon Angela Carter, Sade met « la pornographie au service de la femme, ou, peut-être [...] il la laisse investir par une idéologie ne se trouvant pas aux antipodes de la cause féminine ${ }^{32} »$. The Bloody Chamber n'a été traduit en français qu'en 1985, sous le titre La Compagnie des loups, moins immédiatement sadien que le titre original. Entre l'essai sur Sade et la relecture/réécriture de Perrault parus la même année, il y a un continuum que la préface de Christine Jordis en 1997 met bien en évidence :

Le sujet réel de ces contes, on ne peut s'y tromper, est l'érotisme. [...] Relisant les contes [...] avec le regard du $\mathrm{Xx}^{\mathrm{e}}$ siècle finissant, éclairé par la psychanalyse, corrigé par le féminisme, elle a su les renouveler. [...] Le changement essentiel consiste sans doute à quitter le point de vue de l'homme pour utiliser celui de la femme [...]. Ce sont les femmes qui triomphent. Non seulement en empruntant les armes masculines traditionnelles [...] mais à force d'audace, en refusant d'être une victime terrifiée pour devenir dans l'amour l'égale, la partenaire de l'amant. Ce que démontre l'essai, [...], les contes le mettent en scène. [...] Il suffisait, toute peur étant surmontée, de reconnaître les impulsions profondes venues du désir et de les accepter, dussent-elles inclure le goût de l'humiliation et de la souffrance. Il suffisait d'emprunter, pour se voir, le regard du bourreau [...], de s'ouvrir à la part nocturne de soi-même, cessant ainsi de subir une violence là où il est possible d'éprouver un plaisir ${ }^{33}$.

Ce continuum est sans doute moins évident et moins schématique ou simplement illustratif entre la lecture critique de Sade par Chantal Thomas et sa démarche de création romanesque, comme s'il y avait une forme de pensée propre à l'écriture du roman, tournant autour de cette " blessure » initiale qu'a été la lecture de Sade pour elle. Le roman serait alors, et dans le même geste paradoxal, une façon d'aviver et de soigner « la blessure la

du XVIII ${ }^{\mathrm{e}}$ siècle, Ces choses-là (Paris, POL, 2013, p. 265) : «Laissez-moi cette infinité de choses et de détails, très délicieux selon moi, et qui savent si bien adoucir mes malheurs quand je laisse errer mon imagination, n'espérez pas me changer, toutes ces choses-là et leur ressouvenir sont toujours ce que j'appelle à mon secours quand je veux m'étourdir sur ma situation ».

31. Angela Carter, La Femme sadienne, trad. Françoise Cartano, Paris, Henri Veyrier, 1979. 32. Ibid., p. 68.

33. Angela Carter, La Compagnie des loups [The Bloody Chamber, 1978], trad. Jacqueline Huet, 1985, préface de Christine Jordis, Paris, Seuil, 1997, p. II-III. 
plus rapprochée du soleil », cette définition poétique que René Char donne de la lucidité et qui semble convenir exactement dans le cas de Chantal Thomas, prolongeant peut-être, de façon oblique et indirecte dans son écriture romanesque, la réflexion qu'elle pose dans le titre d'un de ses essais, Comment supporter sa liberté ${ }^{34}$, placé sous le signe de deux «droits de l'homme » revendiqués par Baudelaire dans L'Art romantique : « le droit de se contredire et le droit de $s$ 'en aller $»$.

Anne Coudreuse

Université Paris 13, Sorbonne Paris Cité, Pléiade

34. Chantal Thomas, Comment supporter sa liberté, Paris, Payot et Rivages, 1998. 